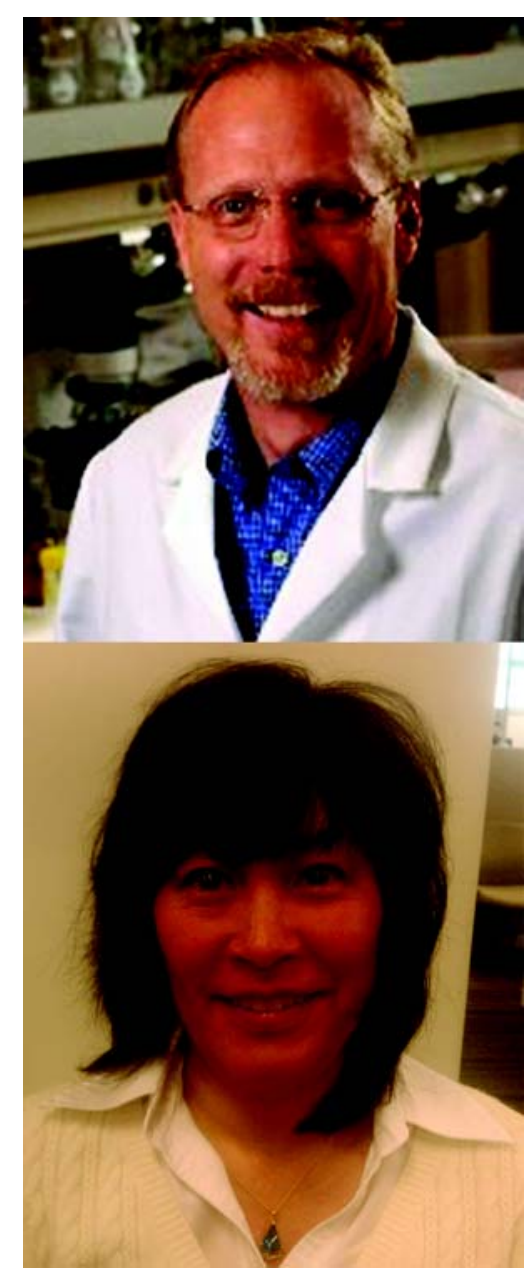

Theo Palmer, PhD; Audrey Kusiak, PhD

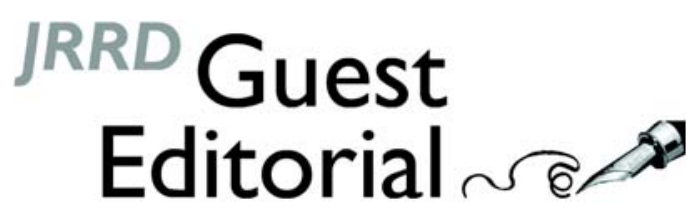

\section{Team presentations from the Route 28 Summits in Neurobiology at the International Symposium on Neural Regeneration}

The International Symposium on Neural Regeneration (ISNR) is a longstanding series of conferences focused on the scientific challenges related to the injured nervous system and the restoration of function. The symposium is the premiere venue for regeneration research, attracting the top national and international researchers in the field. The forum is small, facilitating discussions between attendees and thus creating an ideal opportunity for students and postdoctoral fellows to interact with the leaders in the field. In 2011, the ISNR included the "Route 28 Summits in Neurobiology,” a workshop designed to foster productive collaborations between teams of graduate students, postdoctoral fellows, and faculty attendees by developing novel, "out of the box" solutions to nervous system regeneration-related clinical problems (http://www.route28.org/about-route28.htm).

At the inaugural union of the ISNR and the Route 28 Summits in Neurobiology, three teams of students and postdoctoral fellows were challenged to come up with a solution to the theme "Novel Methods to Exploit Stem Cells for Recovery of Human Central Nervous System Function.” Participants were selected on a first- come, first-served basis upon registration at the Route 28 site. Three groups of four participants were formed and introduced to ISNR attendees. Participants had 3 days to brainstorm within the group, as well as with faculty mentors, conference speakers, and attendees. Information obtained from the lectures and discussions was then synthesized by the teams toward the development of a solution for the recovery of human central nervous system function. Route 28 teams presented their solutions at the final session of the ISNR. Members of the audience voted on the best presentation and the members of the winning team were presented with a cash award. In addition to the oral presentation, Route 28 teams were encouraged to write their solutions into short communications.

The union of the ISNR and Route 28 has resulted in a unique forum where the past, present, and future of regenerative and restorative approaches coexist to provide a fertile ground for collaboration while serving as a platform for young neuroscientists to interact with senior researchers in the field. Several points of discussion regarding this union come to mind. Teams were collaborative and developed innovative solutions despite the time constraints involved. They were able to interact with faculty, speakers, and attendees, who helped formulate hypothesis-based studies to answer the question posed. Moreover, months after the conclusion of the symposium, team members continued working on their submissions to the Journal of Rehabilitation Research and Development, a goal of the original Route 28.

Although teams were smaller in size and number than the traditional Route 28 groups (5 teams of 9 student participants), Route 28/ISNR participants 
were exposed to broader topics areas and were supported by faculty, speakers, and attendees with a wider range of views and backgrounds. The original Route 28 was centered primarily on cell-based therapies, whereas the ISNR exposed its attendees to alternative approaches to restoration of function, including neural, bioengineering, and rehabilitative approaches. It is becoming increasingly clear that a combination of approaches will be necessary to restore function to the injured nervous system. Thus, exposure to these complementary approaches gave the teams a "big picture" view of the nature of regeneration as a multidisciplinary problem.

Lastly, Route 28 participants were introduced to some of the negative aspects of science, including the trials and tribulations of therapies that appeared promising but failed in clinical trials and the lack of reproducibility of preclinical results. The process of translation is not easily mastered, nor is it readily discussed in many scientific forums. We hope that by exposing young investigators to the issues related to translation early in their careers, they will learn from the examples and lessons of the successes and failures and be better prepared when it is their turn to advance scientific results from the bench to the bedside.

In conclusion, we will continue to refine the integration of Route 28 as part of the ISNR because this is a learning process for everyone-organizers and participants. Feedback gathered from the participants was very positive, citing the valuable experience obtained from a team-based approach with guidance from the top researchers in the field of neuroscience. It is in the spirit of Route 28 to challenge its participants to think outside of the box and use a team-based approach to solve a problem in neural regeneration. As one of the participants stated in his review of Route 28 at the ISNR, “. . . our ideas have some basis in reality and could actually inspire actual studies. Some good science has come from 'crazy' ideas before." We hope that those of you who read the entries take this message to heart and are inspired by our next generation of neuroscientists.

Included next are the titles, authors, and abstracts of the three proposals. The full text for each is located in the Appendix (available online only).

\section{Theo Palmer, PhD; ${ }^{1}$ Audrey Kusiak, PhD ${ }^{2 *}$}

${ }^{1}$ Department of Neurosurgery, Stanford University, Palo Alto, CA; ${ }^{2}$ Office of Research and Development, Department of Veterans Affairs, Washington, DC

*Email: audrey.kusiak@va.gov

\section{ACKNOWLEDGMENTS}

Funding/Support: This material (guest editorial and proposals) was based on work supported by the California Institute of Regenerative Medicine, National Institute of Neurological Disorders and Stroke, Paralyzed Veterans of America, Mission Connect, International Neurochemistry Society, and Department of Veterans Affairs.

Additional Contributions: We would like to thank Christopher Moore, $\mathrm{PhD}$, for his helpful comments and suggestions in reviewing the manuscript.

This article and any supplementary material should be cited as follows:

Palmer T, Kusiak A. Team presentations from the Route 28 Summits in Neurobiology at the International Symposium on Neural Regeneration. J Rehabil Res Dev. 2012;49(10):vii-x.

http://dx.doi.org/10.1682/JRRD.2012.12.0224

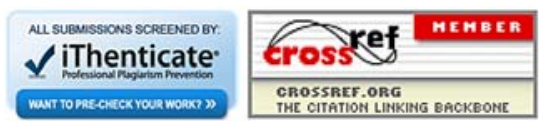

\section{Combining biological and electrical stimulation therapies following spinal cord injury}

Abstract-The following was completed as part of the 2011 Route 28 Summit at the International Symposium on Neural Regeneration. The topic of the Route 28 Summit was "Novel Ways to Exploit Stem Cells for Recovery of Human Central Nervous System Function.” In response to the Route 28 challenge, we propose a novel combinatorial treatment approach using multiple biological 
interventions in conjunction with controlled electrical stimulation to enhance the benefits of a cellular replacement strategy. Using an aligned polymer scaffold seeded with embryonic neural stem cells, we aim to create a relay for the disconnected axons in a transection rodent model of spinal cord injury. This approach will be implemented with (1) a growth factor gradient, (2) chondroitinase $\mathrm{ABC}$ (chABC) injections, and (3) functional electrical stimulation (FES) and in siturecording. We hope to create an environment that is supportive for stem cell survival and differentiation to facilitate neural relays, long distance host axonal regeneration, and functional recovery.

Peggy Assinck, BSc; Basem I. Awad, MD; Roberto Fiorelli, MSc; Bradley Lang, BSc

\section{Stem cell derived radial glial cells in magnetically aligned scaffold for repair after spinal cord injury}

Abstract-The following was completed as part of the 2011 Route 28 Summit at the International Symposium on Neural Regeneration. The topic of the Route 28 Summit was "Novel Ways to Exploit Stem Cells for Recovery of Human Central Nervous System Function.” Traditional cell transplant strategies have not been successful in promoting robust functional recovery following spinal cord injury (SCI). Part of the reason why cell transplant therapies have failed may be related to improper targeting of regenerating axons through the lesion. In this report, we propose a novel therapeutic approach using stem cell-derived radial glial cells in combination with a magnetically aligned fibrin scaffold for targeted axonal regeneration. Animals with a cervical dorsal column injury will receive an injection of embryonic stem cell-derived radial glial cells along with a fibrin-thrombin solution directly into the lesion. The fibrin scaffold will be aligned in situ using magnetic resonance imaging (MRI) prior to polymerization. To further promote axon growth through the lesion, cyclic adenosine monophosphate and chondroitonase $\mathrm{ABC}$ (chABC) will be co-administered rostral and caudal to the lesion. Functional recovery will be evaluated using electrophysiological assessments, and the sticker removal and forelimb reaching tasks. Growth of the regenerating or sprouting host axons will be examined at 3 and 6 mo after injury using MRI and diffusion tensor imaging (DTI). Histological analyses will be conducted to visualize the underlying circuitry mediating putative functional recovery. It is hypothesized that the fibrin-mediated linear organization of the radial glial cells in the lesion will enhance targeted axon outgrowth and improve functional recovery following SCI.

Justin A. Beller, PhD; Jacquelyn Cragg, MPH; Zin Khaing, PhD; Dylan McCreedy, BS

\section{Using genetically modified stem cells to halt the progression of ALS}

Abstract-The following was completed as part of the 2011 Route 28 Summit at the International Symposium on Neural Regeneration. The topic of the Route 28 Summit was, "Novel Ways to Exploit Stem Cells for Recovery of Human Central Nervous System Function.” Amyotrophic lateral sclerosis (ALS) is a neurodegenerative disease characterized by the loss of motor neurons leading to paralysis and death. The vast majority of ALS cases are idiopathic; however, at least $2 \%$ are caused by mutation of the copper-zinc superoxide dismutase 1 gene on chromosome 21. Here, we propose a three-pronged approach: (1) identify the molecular trigger for the onset of symptomatic ALS using a microray approach, (2) develop a genetically modified cell-based treatment, and (3) restore lost respiratory function once disease progression has been halted by an implanted stem cell treatment.

Francisco D. Benavides, MD; Teresa A. Evans, BS, BA; Todd E. White, PhD; Zijia Zhang, BS 
